\title{
Modulation of Memory Formation by Stimulus Content: Specific Role of the Medial Prefrontal Cortex in the Successful Encoding of Social Pictures
}

\author{
Philippe-Olivier Harvey $^{1}$, Philippe Fossati ${ }^{2}$, and Martin Lepage ${ }^{1}$
}

\begin{abstract}
It is unclear whether the involvement of the medial prefrontal cortex (mPFC) during encoding is restricted to the evaluative processing of to-be-encoded stimuli or if it is instead actively engaged during memory formation. The difficulty of assessing the contribution of the MPFC to encoding based on previous neuroimaging studies partly arises from the use of several types of stimuli, such as emotional or social ones. These different types of stimulus content could differently modulate mPFC activity during memory formation and thus partly explain the variable contribution of this region to encoding. Using emotional/neutral and social/nonsocial pictures,
\end{abstract}

\section{INTRODUCTION}

The contribution of the prefrontal cortex (PFC) to episodic memory encoding has been studied extensively in the last decade and the primary focus of much of this work has been directed toward the PFC's dorsolateral and ventrolateral regions (Achim \& Lepage, 2005; Habib, Nyberg, \& Tulving, 2003; Fletcher \& Henson, 2001; Yancey \& Phelps, 2001; Buckner, Kelley, \& Petersen, 1999; Kelley et al., 1998; Wagner, Poldrack, et al., 1998; Tulving, Kapur, Craik, Moscovitch, \& Houle, 1994). Interestingly, the medial PFC (mPFC) has recently received increasing attention in light of several findings implicating this region in memory formation. For instance, some researchers have suggested that the mPFC plays a direct role in emotional associative learning (Laviolette, Lipski, \& Grace, 2005), self-referential encoding (Macrae, Moran, Heatherton, Banfield, \& Kelley, 2004), and socially oriented encoding (Mitchell, Macrae, \& Banaji, 2004). However, other investigators have not found any significant mPFC activation during encoding (Erk, Martin, \& Walter, 2005) or have proposed that this region relates more to the capacity to evaluate future encoding success instead of being directly involved in memory formation (Kao, Davis, \& Gabrieli, 2005).

\footnotetext{
${ }^{1}$ McGill University, Québec, Canada, ${ }^{2}$ Pitié-Salpêtrière Hospital, Paris, France
}

we conducted an event-related functional magnetic resonance imaging study using a subsequent memory paradigm as the main analytical strategy. We observed that the brain activity in the dorsal and orbital mPFC is significantly and specifically predictive of the successful encoding of social compared with nonsocial pictures. In contrast, the activity in the amygdala specifically predicts the successful encoding of emotional compared with neutral pictures. The modulation of the mPFC by social information in a memory encoding context could be associated with the initiation of self-referential processes whose contribution is to enhance memory formation.

Hence, it is unclear whether the involvement of the mPFC during encoding is restricted to an evaluative processing of to-be-encoded stimuli or if it is instead actively engaged in memory formation.

The difficulty of assessing the mPFC's contribution to memory encoding based on previous neuroimaging studies arises from the use of several types of stimuli, ranging from faces (Sergerie, Lepage, \& Armony, 2005), to objects (Achim \& Lepage, 2005), or to complex scenes (Dolcos, LaBar, \& Cabeza, 2004a). Two types of stimulus content in particular have been associated with significant $\mathrm{mPFC}$ activation during picture viewing: emotional stimuli (Phan, Wager, Taylor, \& Liberzon, 2002) and social stimuli (Adolphs, 1999). These two types of stimulus content could differently modulate the mPFC during memory formation and thus partly explain the variable contribution of this region to encoding, as observed in past studies. In the present study, we tested the specific effect of these two types of stimulus content on brain activity during encoding. The innovative aspect of our protocol was to manipulate the social content within the to-be-encoded stimuli while maintaining the emotional content constant across stimuli and vice versa. Based on recent studies (Norris, Chen, Zhu, Small, \& Cacioppo, 2004; Geday, Gjedde, Boldsen, \& Kupers, 2003), we defined social pictures as those showing at least one human being, whereas the nonsocial ones were characterized by the absence of any human 
being. Emotional pictures consisted of a set of stimuli that were previously rated in the positive or the negative valence category in a pilot study not described in this article. The majority of our pictures with social content show more than one human being and involve social interactions between two or more persons. Using these emotional/neutral and social/nonsocial pictures, we conducted an event-related functional magnetic resonance imaging (fMRI) study using a subsequent memory paradigm as the main analytical strategy (Paller \& Wagner, 2002). This approach is the best method for distinguishing brain regions involved in memory formation (i.e., social pictures-hits vs. social pictures-misses) from those involved in stimulus perception/processing (i.e., social pictures vs. nonsocial pictures). Based on previous studies (Sergerie et al., 2005; Dolcos, LaBar, \& Cabeza, 2004b; Mitchell et al., 2004), we hypothesized that the mPFC would be specifically involved in the successful encoding of social compared with nonsocial pictures. We predicted that the encoding of emotional compared with neutral pictures would be associated instead with significant activation of the amygdala.

\section{METHODS}

\section{Subjects}

Seventeen right-handed healthy volunteers (eight women and nine men; mean age $=25$ years; years of education $=17$ ) were recruited for this study. Subjects reported no history of psychiatric or neurological disorders, no history of drugs or alcohol abuse, and were not taking any psychotropic medications at the time of testing. The study was approved by the Montreal Neurological Hospital and Institute Ethics Committee, and informed written consent was obtained from all participants.

\section{Stimuli}

Two hundred forty color pictures were used in the experiment. These pictures were classified into six categories ( 40 pictures in each category): social content with a positive valence (i.e., pleasant events involving one or several persons), social neutral (events involving one or several persons and without apparent emotion), social negative (mostly sad events involving one or several persons), nonsocial positive (mostly beautiful landscapes), nonsocial neutral (household objects such as a fork or an umbrella), and nonsocial negative (scenes of destruction, guns, threatening animals). Pictures were selected from various sources, such as the International Affective Picture System (IAPS) (Lang, Greenwald, Bradley, \& Hamm, 1993), the Empathy Picture System (EPS) (Geday et al., 2003), and from a series of pictures selected from our own collection. We measured the mean luminance for each picture category using CorelDraw software (Corel Corp., Ottawa, Canada). The luminance is basically the density of luminous intensity or the amount of light emitted from a particular area. There was no significant difference between the six groups of pictures regarding luminance $[F(5,240)=$ $0.21, p=.96]$. Social pictures were matched on social complexity [i.e., the mean number $( \pm S D)$ of persons seen on the picture; social neutral $=2.5 \pm 1.7$, social negative $=2.4 \pm 1.4$, social positive $=3.1 \pm 1.1$; main effect of valence regarding social complexity, $F(2,120)=$ $2.12, p=.11]$. Because all picture stimuli from the IAPS and EPS were previously matched for general color composition and we included approximately the same number of these pictures in each stimulus category, we assumed that general color composition was matched across our experimental conditions. To avoid the potential confounding effect of arousal over emotional valence during encoding processes or a potential arousal level difference between social and nonsocial pictures, we excluded pictures known to trigger high arousal (based on previous pictures rating from the IAPS), such as pictures presenting erotic scenes or mutilated bodies. Finally, a seventh category made of 40 control pictures was added. Twenty social and 20 nonsocial pictures were randomly selected, and a series of transformations was applied (blur, pixelization, horizontal, and vertical inversion) using Adobe Photoshop 7.0 (Adobe Systems, San Jose, $\mathrm{CA})$. Thus, 40 unrecognizable control pictures were obtained, matched for luminance and general color composition. These control pictures were included in our paradigm to provide a general overview of the brain regions involved in the viewing of complex pictures relative to abstract pictures.

\section{Experimental Design}

The experiment was divided in two parts: (1) an encoding phase and (2) a recognition test. In the encoding phase, 140 pictures from pregenerated list I or II (counterbalanced across subjects) (20 from each category, including control pictures) were pseudorandomly presented to the subjects, one at a time. Each picture was presented for $3 \mathrm{sec}$, followed by a fixation crosspresented for 2 sec. For each picture presentation, subjects were required to identify the presence of a human being within the picture by clicking on predetermined "yes" or "no" buttons. To maximize the brain activation associated with the viewing of the pictures, subjects were not explicitly told to learn or encode the pictures. Subjects were told to press anything when a control picture appeared. One of two different sets of pictures counterbalanced across subjects was presented in the encoding phase. The encoding phase was followed by a recognition test. Two hundred eighty pictures were presented for the recognition test, including 
140 previously seen or old pictures and 140 new ones. As for the encoding phase, each picture was presented for $3 \mathrm{sec}$, followed by a fixation cross presented for $2 \mathrm{sec}$. Subjects were asked to identify the "old" pictures from the new ones by clicking on predetermined "old" or "new" buttons. The recognition test was conducted over two sessions of 140 pictures ( 70 old pictures and 70 new ones). Because the main focus of the present article is memory formation, we only report the fMRI data concerning the encoding phase.

After the scanning session, participants performed an emotional picture-rating task on a laptop computer. To get a more precise and idiosyncratic rating from subjects, each picture was accompanied by a continuous line with the label "very negative" at the left end of the line and the label "very positive" at the right end of the line. Subjects were further told that the middle of the line was associated with neutrality. Using a mouse, subjects moved an arrow on this line and clicked the left button once the arrow was well positioned on the line according to the emotional valence of the picture. This procedure indicated their personal emotional valence rating for each stimulus presented. The continuous line was in fact an ordinal scale ranging from 1 (very negative) to 323 (very positive). Mean valence values $( \pm S D)$ for each condition were the following: nonsocial neutral $=163 \pm 3.6$, nonsocial negative $=91 \pm 23.3$, nonsocial positive $=239 \pm 32.3$, social neutral $=170 \pm$ 16.6, social negative $=68 \pm 20.6$, and social positive $=$ $261 \pm 28.9$. Mean valence values for combined neutral $(167 \pm 18.7)$, negative $(79 \pm 29.3)$, and positive $(249 \pm$ 18.1) pictures differed significantly among themselves [main effect of valence, $F(2,240)=1142.79, p<.001$; Bonferroni $=$ negative $<$ neutral $<$ positive $]$. Moreover, the emotional valence was not significantly different between social $(169 \pm 83.1)$ and nonsocial pictures $(166 \pm 63)$ [main effect of content, $F(1,240)=0.07$, $p=.787]$.

\section{Data Acquisition}

fMRI data were acquired at the Montreal Neurological Institute on a 1.5-T Siemens (Berlin, Germany) Sonata whole-body system equipped with a standard head coil, using gradient EPI sequences. A vacuum cushion stabilized the subject's head. Stimuli were generated by an IBM PC laptop computer running E-PRIME (Psychology Software Tools, Pittsburgh, PA) and projected via a LCD projector and mirror system. A mouse connected to the computer recorded the subject's responses. Functional T2*-weighted images were acquired with blood oxygenation level-dependent (BOLD) contrast (three functional runs of 278 volumes, $\mathrm{TR}=2550 \mathrm{msec}, \mathrm{TE}=50 \mathrm{msec}$, flip angle $=90^{\circ}$, field of view $=256 \mathrm{~mm}$, matrix $=64 \times$ 64), covering the entire brain (30 interleaved slices parallel to the anterior-posterior commissural plane; in plane resolution $=4 \times 4 \mathrm{~mm}$; thickness $=4 \mathrm{~mm})$. After the functional session, a high-resolution T1-weighted anatomical volume was acquired using a gradient-echo pulse sequence $(22 \mathrm{msec}, \mathrm{TE}=9.2 \mathrm{msec}$, flip angle $=$ $30^{\circ}$, voxel size $=1 \times 1 \times 1 \mathrm{~mm}$ ).

\section{Data Analysis}

fMRI data were analyzed using Statistical Parametric Mapping (SPM2; Wellcome Department of Cognitive Neurology, London, UK). Image preprocessing was done according to a standard procedure (Ashburner \& Friston, 1997). Briefly, images were time corrected to account for differences in sampling times for different slices, realigned to the first volume to correct for interscan movement, and spatially normalized (voxel size $=$ $2 \times 2 \times 2 \mathrm{~mm}$ ) to the standard space of Talairach and Tournoux using the Montreal Neurological Institute template (Collins, Neelin, Peters, \& Evans, 1994). Finally, the images were smoothed with an isotropic 8-mm full width half-maximum Gaussian kernel. Low-frequency temporal drifts were removed by applying a high-pass filter. Data were analyzed using the general linear model, in which individual events were modeled by a synthetic hemodynamic response function. For the encoding part of this study, three main analyses were conducted. In the first analysis, four event types were defined, based on the pictures' social content (social and nonsocial) and emotional context (neutral and emotional), as well as a fifth event type representing the control pictures. This first analysis allowed us to compare the brain activity associated with the viewing of the pictures at the encoding phase, that is, to identify the regions associated with the evaluative processing of emotional/ social pictures compared with their respective neutral/ nonsocial control pictures. We then performed analyses for the subsequent memory effect (SME). The SME analyses were conducted using the subjects' performance rates obtained in each condition during the memory recognition test. Two distinct analyses were performed for the SME: The first one was based on the social content of the picture (four event types: nonsocial "subsequently remembered or hits," nonsocial "subsequently forgotten or misses," social "hits," and social "misses"), and the second one was based on the emotional context (four event types based on the emotional conditions [neutral and emotional] and on the performance during recognition memory [hits and misses]). The number of forgotten pictures during the recognition test was very low for some subjects. Thus, some subjects have provided only two or three events for some pooled conditions/event types. We set a minimum cutoff of 10 events per pooled condition (i.e., at least 10 pictures had to be forgotten for the pooled neutral condition, which included social and nonsocial neutral pictures). Subjects that did not have enough 
subsequently forgotten trials during encoding were not analyzed. Five of 17 subjects were excluded from SME analyses once this cutoff was applied. Linear contrasts of subject-specific parameter estimates for conditions of interest were generated and taken to a second-level random effects model. The threshold was set at $p<.001$ (uncorrected) for the first analysis and at $p<.001$ (one sample $t$ tests) and $p<.005$ (uncorrected) (paired $t$ tests) for the SME analyses.

To test the specific role of the amygdala in the successful encoding of emotional compared with neutral pictures and the specific role of the mPFC in the successful encoding of social compared with nonsocial pictures, we conducted two paired $t$ tests: (1) (emotional hits vs. emotional misses) minus (neutral hits vs. neutral misses); (2) (social hits vs. social misses) minus (nonsocial hits vs. nonsocial misses). Inclusive masks consisting only of regions significantly involved in the successful encoding of emotional pictures or social pictures were used in the paired $t$ tests. These inclusive masks were obtained from the contrasts (one-sample $t$ tests, $p<.005$ uncorrected) "emotional hits minus emotional misses" and "social hits minus social misses" and were drawn using MRIcro software. Thus, the paired $t$ tests identified the regions that were significantly more active in the successful encoding of emotional/social pictures than during the successful encoding of neutral/nonsocial pictures.

Finally, the parameter estimates of the BOLD signal observed in the peak voxel of the target regions obtained from the paired $t$ tests were calculated for eight conditions of interest: (1) social hits, (2) social misses, (3) nonsocial hits, (4) nonsocial misses, (5) emotion hits, (6) emotion misses, (7) neutral hits, and (8) neutral misses. Using parameter estimates of all 12 subjects for all eight conditions, we conducted two distinct repeated measures analyses of variance (ANOVAs) (two levels within-subjects factors: memory factor [hits and misses] and content factor [social and nonsocial or emotional and neutral]) for each target region (thus, six distinct repeated-measures ANOVAs overall). Because SPM paired $t$ tests are strictly for calculating a significant difference between two paired groups of contrasts, these BOLD signal analyses were added to test the more general main effects of memory and content on the brain activation for each target region. Moreover, the peak voxel of each target region was selected from a specific comparison, either ([social hits vs. social misses] vs. [nonsocial hits vs. nonsocial misses]) or ([emotional hits vs. emotional misses] vs. [neutral hits vs. neutral misses]). It was then important to test the BOLD signal for the "other comparison" and thus to clarify whether the absence of a significant difference in the target region for this latter comparison is explained by (1) an absence of modulation by encoding for both content conditions or (2) a general involvement in encoding for both content conditions.

\section{RESULTS}

\section{Behavioral Performance}

Performance during the encoding task (to determine the presence of a human being in the picture) was almost perfect for each subject at each condition. Only the nonsocial neutral pictures were not perfectly classified, with 99\% accuracy. Moreover, no significant difference was observed in reaction time between content conditions (social vs. nonsocial) $[F(1,16)=2.86, p=.11]$ or among emotional conditions (positive, negative, and neutral) $[F(2,15)=3.19, p=.07]$. There was no significant interaction between content and emotion regarding reaction time $[F(2,15)=1.83, p=.17]$. During the recognition phase, the hit rate (used for SME analyses) did not significantly differ either between content conditions $[F(1,16)=0.43, p=.52]$ or between emotional conditions $[F(2,15)=0.90, p=.43]$ (nonsocial neutral, $86 \%$ of remembered pictures; nonsocial negative, $88 \%$; nonsocial positive, $84 \%$; social neutral, $85 \%$; social negative, $85 \%$; social positive, $85 \%$ ). There was no significant interaction between content and emotion regarding the hit rate at the recognition phase $[F(2,15)=$ $1.71, p=.22]$. The mean percentage of hits (all conditions pooled together) obtained at the recognition phase by the 12 subjects included in the SME analyses was $82 \%$ (the worst individual performance mean was $72 \%$ of hits) compared with $92 \%$ for the five subjects excluded from the SME analyses.

\section{Functional Magnetic Resonance Imaging Results}

\section{Picture Viewing}

Before subsequent memory analyses, we first examined the brain activation specifically associated with the viewing (perceptive/evaluative processing) of social compared with nonsocial pictures and with the viewing of emotional (both positive and negative valence) compared with neutral pictures. The main regions showing activation associated with social picture viewing were located in the dorsal and ventral mPFC [Brodmann's areas (BA) $10 / 11, t=7.71]$, the hippocampi $(t=7.46)$, the right fusiform gyrus (BA 37; $t=6.90$ ), the posterior cingulate (BA 31; $t=7.11$ ), the bilateral superior and middle temporal gyri (BA 37/38; $t=10.59$ ), and the cuneus bilaterally (BA $18 ; t=9.17$ ). The reverse contrast (nonsocial vs. social) showed activations in the left middle frontal gyrus (BA 9; $t=4.07$ ), the precuneus bilaterally (BA $7 ; t=8.65$ ), the bilateral inferior temporal gyri (BA 20; $t=5.56$ ), the bilateral superior occipital gyri (BA 19; $t=7.16)$, and the cerebellum $(t=9.15)$. On the other hand, the emotional pictures specifically activated the mPFC (BA 9/10; $t=5.03$ ), the left insula (BA 13; $t=5.40)$, the left amygdala $(t=3.04)$, the right ventrolateral PFC (BA 11; $t=4.22$ ), the right fusiform gyrus (BA 37; $t=9.05$ ), and the cuneus bilaterally (BA $18 ; t=$ 7.57) compared with the neutral pictures. The reverse 
contrast (neutral vs. emotional) showed activations in the right inferior frontal gyrus (BA $11 ; t=4.59$ ), the right inferior temporal gyrus (BA 20; $t=5.28$ ), the right inferior parietal lobule (BA 40; $t=4.66$ ), and the left fusiform gyrus (BA 37; $t=4.91$ ) (see Figure 1 ; all previous activations exceeded $p<.001$ on the single voxel level; $16 \mathrm{dF}$ ).

\section{Subsequent Memory Effect}

Using the contrast social hits versus social misses, we identified brain regions that were statistically predictive of the successful encoding of social pictures. These regions are the dorsomedial PFC bilaterally, the left ventrolateral PFC, the inferior frontal gyri bilaterally, the parahippocampal gyri, and the fusiform gyri bilaterally. The nonsocial hits versus nonsocial misses contrast was also performed and showed significant activations in the orbitofrontal and inferior (dorsolateral) frontal gyri bilaterally, the left hippocampus, the right parahippocampal gyrus, and the fusifom gyri bilaterally (see Table 1 for details).

Regions associated with the successful encoding of the emotional and neutral pictures were determined using the contrasts emotional hits versus emotional misses and neutral hits versus neutral misses. Successful encoding of the emotional pictures was associated with the activation of the superior and inferior frontal gyri, the amygdala bilaterally, the right parahippocampal gyrus, and the fusifom gyri bilaterally. The neutral pictures that were subsequently remembered during the recognition test were related to a greater activation of the left dorsal PFC, the right superior temporal gyrus, the left parahippocampal gyrus, and the fusiform gyri bilaterally compared with the subsequently forgotten neutral pictures (see Table 1 for details).

Paired $t$ tests were conducted to identify brain regions specifically involved in the successful encoding of emotional compared with neutral pictures (contrast: [emotional hits vs. emotional misses] minus [neutral hits vs. neutral misses]) and social compared with nonsocial pictures (contrast: [social hits vs. social misses] minus [nonsocial hits vs. nonsocial misses]). Two bilateral regions were significantly more involved in the successful encoding of emotional compared with neutral pictures: the amygdala $(t=4.44)$ and the fusiform gyri (BA 37; $t=5.07$ ) (see Figure $2 \mathrm{~A}$ ). Three main regions appeared to be specific to the successful encoding of social pictures compared with the nonsocial ones: the right dorsomedial PFC (BA 10; $t=3.37$ ), the orbital PFC and $\mathrm{mPFC}(\mathrm{BA} 11 ; t=4.95)$, and the left superior frontal gyrus (BA $8 ; t=5.70$ ) (see Figure 2B).

\section{Parameter Estimates of the Blood Oxygenation Level-dependent Signal}

The specificity of the amygdala for the successful encoding of emotional content and the specificity of the dorsal and orbital mPFC for the successful encoding of social content were also tested by calculating the parameter estimates of the BOLD activation observed in the peak voxel of each of these three regions (obtained from the paired $t$ tests). For the dorsomedial PFC (peak voxel $=$ 10, 56, 32), the repeated measures ANOVA comparing social and nonsocial conditions yielded a significant main effect of memory (hits vs. misses) $[F(1,11)=$ $6.20, p=.03]$ and did not yield a significant main effect of content (social vs. nonsocial) $[F(1,11)=0.15, p=.70]$. A significant Memory $\times$ Content interaction was also observed $[F(1,11)=12.28, p=.005]$, suggesting that a greater BOLD signal in the dorsomedial PFC was specific to the condition "social hits" (Figure 3A). Concerning the comparison between emotional and neutral conditions, a significant main effect was observed for the memory factor $[F(1,11)=9.28, p=.01]$, thus emphasizing the role of the dorsomedial PFC in encoding. However, no significant effect was observed either for the content factor $[F(1,11)=1.45, p=.25]$ or for the Memory $\times$ Content interaction $[F(1,11)=0.33, p=.58]$ (Figure $3 \mathrm{~B}$ ). This result suggests that the BOLD signal in the dorsomedial PFC was not significantly modulated by
Figure 1. Brain activation during the viewing of emotional pictures (left) compared to neutral ones (contrast: all emotional pictures vs. all neutral pictures) and during the viewing of social pictures (right) compared to nonsocial ones (contrast: all social pictures vs. all nonsocial pictures). Statistical parametric maps (one sample $t$ test, 17 subjects analyzed) show significant activation at a statistical threshold of $p<.001$ (uncorrected) with 10 voxels as the minimal cluster size.

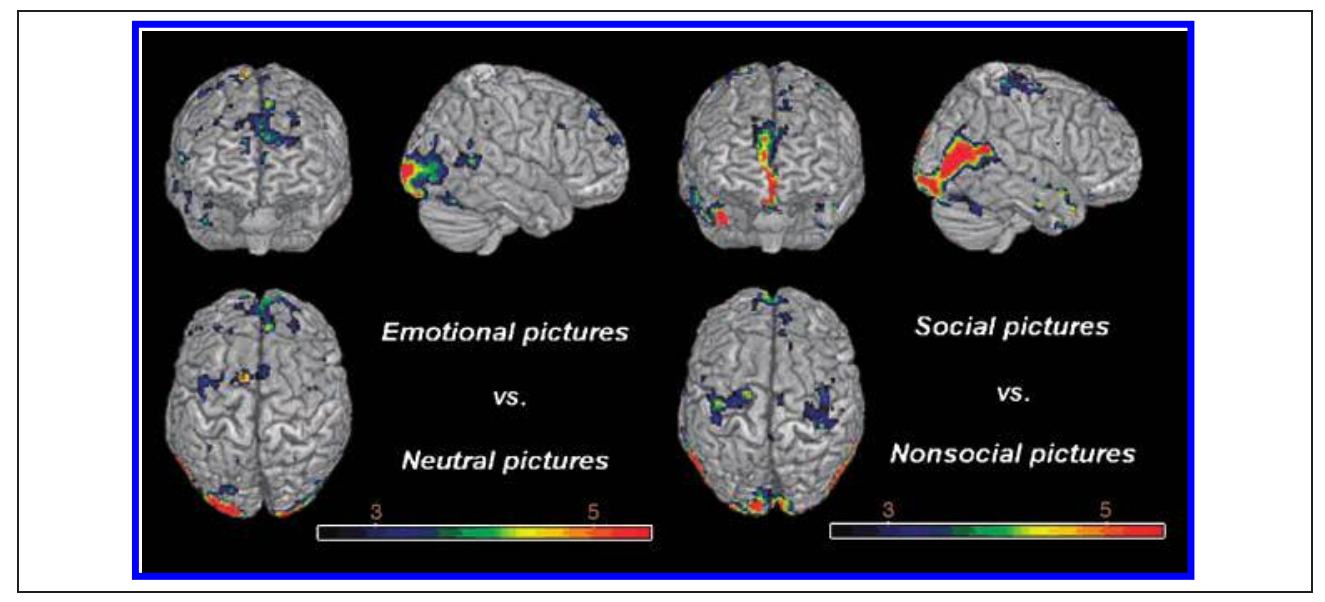


Table 1. Brain Activation Associated with the Successful Encoding (Subsequently Remembered [Hits] vs. Subsequently Forgotten [Misses]) of Social, Nonsocial, Emotional, and Neutral Pictures

\begin{tabular}{|c|c|c|c|c|c|}
\hline \multirow[b]{2}{*}{ Brain Area } & \multirow[b]{2}{*}{ Hemisphere/BA } & \multicolumn{3}{|c|}{ Stereotaxic Coordinates } & \multirow[b]{2}{*}{$t$ Value } \\
\hline & & $x$ & $y$ & $z$ & \\
\hline \multicolumn{6}{|l|}{ Social hits vs. social misses } \\
\hline Dorsal PFC & $\mathrm{L} / 10$ & -18 & 56 & 22 & 5.97 \\
\hline Dorsomedial PFC & $\mathrm{R} / 9$ & 10 & 60 & 34 & 3.84 \\
\hline Ventrolateral PFC & $\mathrm{L} / 47$ & -44 & 38 & -8 & 4.14 \\
\hline Inferior frontal gyri & $\mathrm{L}-\mathrm{R} / 45$ & 60 & 18 & 18 & 4.58 \\
\hline Anterior parahippocampal gyrus & $\mathrm{R} / 35$ & 22 & -6 & -24 & 4.08 \\
\hline Posterior parahippocampal gyrus & $\mathrm{L} / 28$ & -22 & -18 & -22 & 4.79 \\
\hline Fusiform gyri & $\mathrm{L}-\mathrm{R} / 37-19$ & -46 & -72 & -14 & 8.00 \\
\hline \multicolumn{6}{|l|}{ Nonsocial hits vs. nonsocial misses } \\
\hline Middle frontal gyrus & $\mathrm{L}-\mathrm{R} / 11$ & 26 & 34 & -16 & 7.01 \\
\hline Dorsolateral PFC & $\mathrm{L}-\mathrm{R} / 9-44$ & 46 & 12 & 30 & 3.61 \\
\hline Hippocampus & $\mathrm{L}$ & -24 & -16 & -12 & 3.79 \\
\hline Parahippocampal gyrus & $\mathrm{R} / 36$ & 32 & -24 & -26 & 4.90 \\
\hline Fusifom gyri & $\mathrm{L}-\mathrm{R} / 37$ & 46 & -62 & -16 & 7.88 \\
\hline \multicolumn{6}{|l|}{ Emotional hits vs. emotional misses } \\
\hline Superior frontal gyrus & $\mathrm{L}-\mathrm{R} / 11$ & -32 & 36 & -12 & 5.32 \\
\hline Inferior frontal gyrus & $\mathrm{L}-\mathrm{R} / 8-9-46$ & -46 & 18 & 26 & 6.10 \\
\hline Amygdala & $\mathrm{L}-\mathrm{R}$ & 20 & -4 & -20 & 5.23 \\
\hline Parahippocampal gyrus & $\mathrm{L}-\mathrm{R} / 36$ & 30 & -26 & -24 & 7.88 \\
\hline Fusiform gyri & $\mathrm{L}-\mathrm{R} / 37-19$ & -44 & -52 & -14 & 8.24 \\
\hline \multicolumn{6}{|l|}{ Neutral hits vs. neutral misses } \\
\hline Dorsal PFC & $\mathrm{L} / 10$ & -16 & 58 & 22 & 3.46 \\
\hline Superior temporal gyrus & $\mathrm{R} / 22$ & 58 & 6 & 4 & 4.57 \\
\hline Parahippocampal gyrus & $\mathrm{L} / 28$ & -22 & -6 & -28 & 3.60 \\
\hline Fusiform gyri & $\mathrm{L}-\mathrm{R} / 37$ & 48 & -60 & -16 & 6.49 \\
\hline
\end{tabular}

Activations are reported if they exceeded $p<.001$ (uncorrected) on the single voxel level. Stereotaxic coordinates represent the peak height voxel of the cluster.

emotional content during encoding. Concerning the orbital PFC (peak voxel $=-4,38,-26$ ), the repeated measures ANOVA comparing social and nonsocial conditions did not reveal a significant main effect of memory $[F(1,11)=2.27, p=.16]$ or a significant main effect of content $[F(1,11)=0.61, p=.45]$. It did, however, show a significant Memory $\times$ Content interaction $[F(1,11)=$ $22.88, p=.006$ ] (Figure 3C). Again, this result points toward a specific involvement of the orbital PFC for the subsequently remembered social pictures. No significant results were obtained from the comparison between emotional and neutral conditions in the orbital PFC [main effect of memory, $F(1,11)=1.81, p=.21$; main effect of content, $F(1,11)=0.02, p=.90$; Memory $\times$ Content interaction, $F(1,11)=0.70, p=.42]$ (Figure 3D). Like the dorsomedial PFC, the orbital PFC was not significantly modulated by the emotional content compared with neutral content during encoding.

BOLD activation analysis in the amygdala (peak voxel $=$ $18,-6,-18)$ for the comparison between social and 
Figure 2. (A) Main target region (amygdala; peak voxel $=$ $18,-6,-18)$ specific to the successful encoding of emotional pictures compared to neutral ones (contrast: [emotional hits vs. emotional misses] minus [neutral hits vs. neutral misses]). (B) Target regions specific to the successful encoding of social pictures compared to nonsocial ones (dorsomedial PFC, peak voxel $=10,56,32$; orbital PFC, peak voxel $=-4,38,-26$ ) (contrast: [social hits vs. social misses] minus [nonsocial hits vs. nonsocial misses]). Statistical parametric maps (paired $t$ tests, 12 subjects analyzed) show significant activation at a statistical threshold of $p<.005$ (uncorrected) with 10 voxels as the minimal cluster size.

Figure 3. Parameter estimates of the BOLD signal observed in the peak voxel of the dorsomedial PFC (A and B), the orbital PFC (C and D), and the amygdala (E and $\mathrm{F}$ ) for eight conditions of interest (social "hits," social "misses," nonsocial "hits," nonsocial "misses," emotion "hits," emotion "misses," neutral "hits," and neutral "misses"). Peak voxels were obtained from the contrast (social hits vs. social misses) minus (nonsocial hits vs. nonsocial misses) for the dorsomedial and the orbital PFC and from the contrast (emotional hits vs. emotional misses) minus (neutral hits vs. neutral misses) for the amygdala.
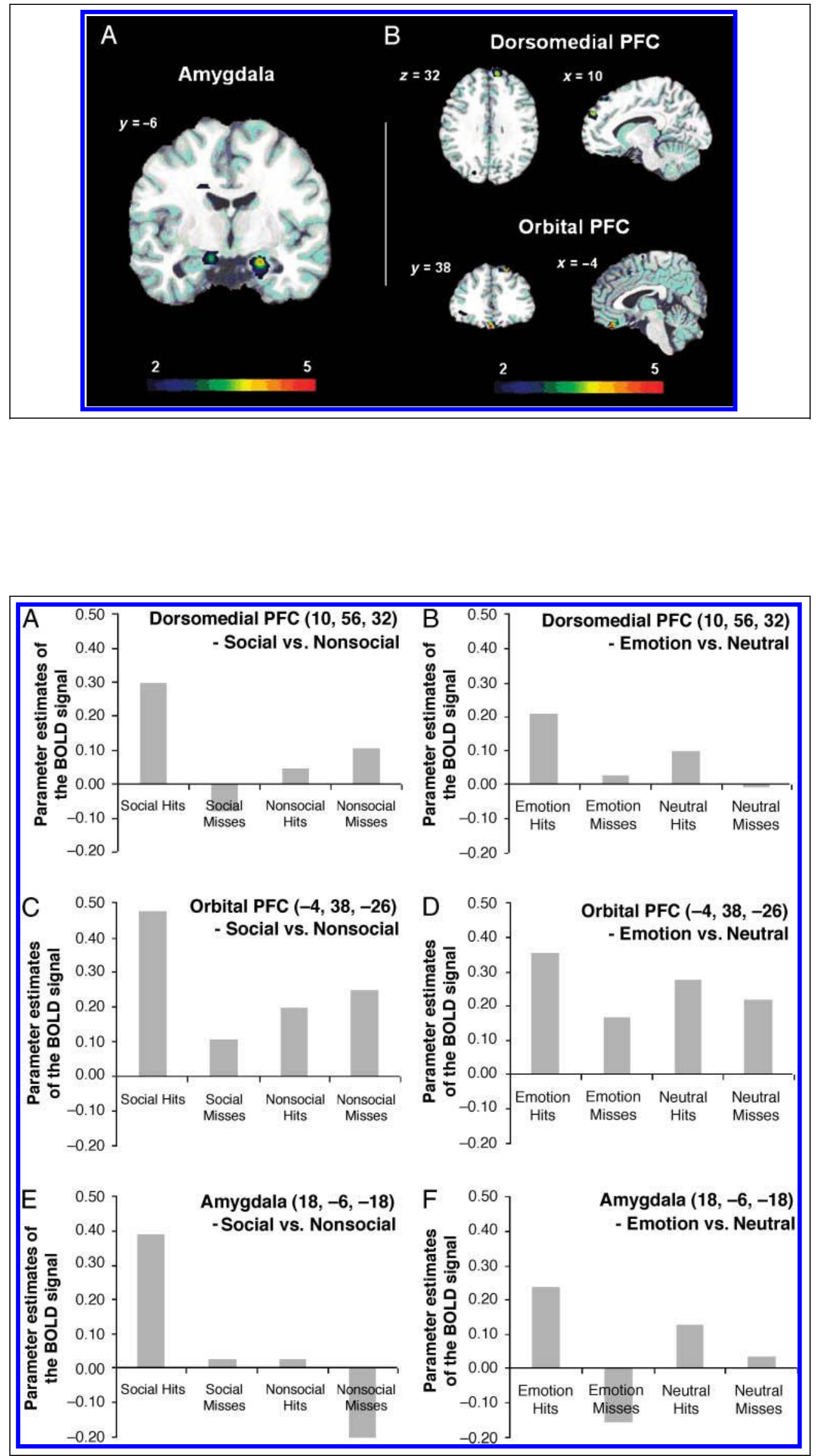
nonsocial conditions revealed significant main effects of memory $[F(1,11)=10.14, p=.009]$ and content $[F(1,11)=13.13, p=.004]$, but no significant Memory $\times$ Content interaction $[F(1,11)=0.96, p=.34]$ (Figure $3 \mathrm{E})$. The significant main effect of memory shows that the amygdala was involved in the encoding of our pictures. Finally, the ANOVA comparing the emotional and the neutral conditions in the amygdala revealed a significant main effect of memory $[F(1,11)=5.84, p=$ $.034]$, but no significant main effect of content $[F(1,11)=$ $0.14, p=.72]$. A significant Memory $\times$ Content interaction $[F(1,11)=7.82, p=.017]$ suggests a greater BOLD signal activation in the amygdala specifically for the emotional hits condition (Figure 3F).

In a post hoc analysis, we carried out a fixed-effect analysis with our 12 subjects. Eight independent conditions were defined: (1) socioemotional hits, (2) socioemotional misses, (3) socioneutral hits, (4) socioneutral misses, (5) nonsocial emotional hits, (6) nonsocial emotional misses, (7) nonsocial neutral hits, and (8) nonsocial neutral misses. Using SPM2 (SPM contrast manager), we calculated the triple interaction using the contrast "all-social hits versus all-social misses" as an inclusive mask (mask at 0.05 uncorrected). Thus, among the brain regions involved in the successful encoding of social pictures, we identified those showing a three-way Social Content $\times$ Emotional Content $\times$ Subsequent Memory interaction, that is, those significantly modulated by social content compared with emotional content during encoding. The only frontal regions showing such threeway interaction were in the superior frontal gyrus: lateral pole $(-28,64,16$; BA $10 ; t=2.30)$ and dorsomedial part (8, 66, 24; BA 10; $t=2.03)$ ( $p=.05$, uncorrected).

\section{DISCUSSION}

We observed that the brain activity in the MPFC is significantly and specifically predictive of the successful encoding of social compared with nonsocial pictures, but is not significantly implicated in the encoding of emotional compared with neutral pictures. Instead, the activity in the amygdala specifically predicts the successful encoding of emotional pictures.

\section{Medial Prefrontal Cortex Contribution to the Successful Encoding of Social Stimuli}

The MPFC is not only involved in the evaluative processing of social stimuli as suggested by the mPFC activation associated with the viewing of social pictures, but it is also actively engaged in the encoding of stimuli with social content. We suggest that social content, compared with nonsocial content, specifically modulates the contribution of the mPFC to episodic encoding.

The mPFC among other regions is a key structure involved in social processing (Gallagher \& Frith, 2003;
Vogeley et al., 2001; Adolphs, 1999). Indeed, recent fMRI studies have demonstrated a major involvement of the mPFC in the judgment of socially relevant stimuli, from faces (Mitchell et al., 2004) to complex social interactions (Iacoboni et al., 2004). Some authors attribute this mPFC activation to the ability to evaluate the psychological state of other persons or oneself (Mitchell, Neil Macrae, \& Banaji, 2005; Kelley et al., 2002). In a recent study supporting the involvement of the mPFC in social encoding, Mitchell et al. (2004) combined statements with faces for two different memory tasks: a socially oriented task (SOT) and a nonsocial task. Using SME analyses, they demonstrated the involvement of the dorsomedial PFC specifically during the SOT. Indeed, Mitchell et al. (2004) extracted the BOLD signal within the dorsomedial PFC and reported a significant $2 \times$ 2 interaction (Memory Task $\times$ Subsequent Memory).

One methodological difference between the study of Mitchell et al. (2004) and the present one is the requirement for explicit complex social processing. Indeed, Mitchell et al.'s SOT-termed impression formation required participants to look at the faces and to use the statements to infer the personality traits of target persons. In another study, Mitchell et al. demonstrated that such SOT is specifically associated with dorsomedial PFC activation. One could argue that this type of socially oriented processing, although contributing to successful encoding when engaged, may not be absolutely necessary to successfully encode social stimuli. Our design used only one simple task in which social content was manipulated within the stimulus set. Thus, our subjects were not required to intentionally evaluate/process the psychological states depicted by the stimuli. In fact, the main conceptual difference between Mitchell et al. and the present study is about the factor that influences mPFC activity. Mitchell et al. manipulated the orienting task (impression formation vs. sequencing task) while the material was held constant. We instead kept the orienting task constant and made minimal demands on social processing while manipulating the presence or absence of people. Our study suggests that explicit complex socially relevant processing is not a necessary condition to observe a modulation of the dorsomedial PFC during memory formation. We found that the presence of people within a complex picture can modulate brain activity in this region. The fact that we found significant involvement of the dorsomedial PFC using a simple encoding task, as opposed to a SOT that triggers the judgment of mental characteristics, raises questions regarding potential implicit socially relevant processing that might be used to successfully encode social content.

The specific contribution of the orbital mPFC to the encoding of social pictures is also consistent with the literature. It has been demonstrated that patients with damage to the orbitofrontal cortex and the ventromedial PFC can have severe deficits in social functioning (Stone, Baron-Cohen, \& Knight, 1998). These patients can still 
express empathy, but they cannot connect their theory of mind inferences to an understanding of emotion. In other words, the orbitofrontal cortex is an essential brain structure for interpreting the valence and the significance of others actions and intentions (Rolls, 1996; Adolphs, Tranel, Damasio, \& Damasio, 1994). Because $66 \%$ of our social pictures also have emotional content, it is possible that the interpretation of the emotional valence extracted from a social context favored the successful encoding of these social pictures and thus has prompted the orbital mPFC activation.

\section{Self-referential Processing and Social Encoding}

The involvement of the mPFC in social processing is complicated by the dual role of this region in both inferring the mental states of others (theory of mind) (Gallagher \& Frith, 2003) and performing tasks that require people to introspect about their own inner mental states (self-referential) (Fossati et al., 2003; Kelley et al., 2002). A functional dissociation has been recently proposed by Schilbach et al., (2005), who have used dynamic virtual characters in fMRI to distinguish the neural correlates of being personally involved in social interaction (self-referential) as opposed to being a passive observer of social interaction between others (theory of mind) (Schilbach et al., 2005). Their results suggest that although the ventromedial PFC is associated with the analysis of social content as accessible in interactionally relevant gestures, differential activation of the dorsomedial PFC subserves the detection of self-reference. Based on these findings, the right dorsomedial PFC activation found in our study could suggest that implicit self-referential processes are part of the social encoding. Such a proposition is consistent with past studies that showed a reliable activation of the right dorsomedial PFC during self-referential encoding (Fossati et al., 2004; Macrae et al., 2004). In a meta-analysis of the selfreferential effect, Symons and Johnson (1997) suggested that self-referential encoding has the potential to provide self-relevant category labels that may enhance subsequent recall by facilitating the reinstatement of encoding conditions at retrieval. Similarly, other researchers refer to this "self-relevant category labeling" as the simulation theory (Gallese \& Goldman, 1998), which postulates that people use their own mental states to explain the mental processes of others. This implicit self-referential strategy to successfully encode pictures might be more automatic for social pictures compared with nonsocial ones and would likely modulate the involvement of the mPFC during social encoding.

\section{Medial Prefrontal Cortex, Amygdala, and Emotional Encoding}

The statistical comparison between the successful encoding of emotional stimuli versus neutral stimuli revealed a significant activation of the amygdala. The contribution of this structure to emotional encoding has been extensively documented in past studies (Dolcos et al., 2004a; Erk et al., 2003; Canli, Zhao, Brewer, Gabrieli, \& Cahill, 2000; Hamann, Ely, Grafton, \& Kilts, 1999). According to the modulation hypothesis, emotional encoding is linked to modulatory influences of the amygdala on encoding processes occurring in medial temporal lobe structures (McGaugh, McIntyre, \& Power, 2002). Our paradigm did not show any significant activation of the mPFC during the encoding of emotional stimuli compared with neutral ones. Thus, our results could suggest that the $\mathrm{MPFC}$ is only involved in an evaluative processing of the emotional information (as suggested by the MPFC activation associated with the viewing of emotional pictures) and is not actively engaged in emotional encoding. A direct statistical comparison between social content and emotional content will be needed to confirm this hypothesis.

Based on our results, it appears that the amygdala plays a more general role in both emotional and social encoding. Indeed, even if the amygdala was not significantly involved in social encoding (paired $t$ test), the post hoc parameter estimates analysis for the social versus nonsocial comparison does suggest a general involvement of the amygdala in the encoding of social pictures: (1) There is a significant main effect of memory, because the amygdala is more active for the subsequently remembered pictures compared with the forgotten ones. (2) There is also a significant main effect of content, because the amygdala is more active for the social pictures compared with nonsocial ones regardless of the encoding success. It is possible that social pictures are automatically processed as being potentially emotional. Indeed, social stimuli are likely the most emotionally evocative stimuli for humans and most brain regions subserving social processing are also implicated in emotional processing (Norris et al., 2004; Geday et al., 2003). Moreover, several studies suggest that the amygdala is implicated in the extraction of emotional meaning from social stimuli, in addition to general emotional processing (Winston, Strange, O’Doherty, \& Dolan, 2002; Adolphs, Tranel, \& Damasio, 1998). These observations underscore the highly dependent link between social and emotional processes.

\section{Caveats and Limitations}

One potential confound associated with the mPFC activation during social stimuli viewing is related to greater attentional demand. Because they are likely more complex, social stimuli may receive greater attention compared with nonsocial ones, and the dorsal mPFC activation could reflect this increase in attention (Bush, Luu, \& Posner, 2000). Using an SME paradigm, we have directly compared social pictures with other social pictures. Reaction times and response accuracy during the encoding task were not statistically different 
between subsequently remembered and forgotten pictures. Thus, we can safely assume that the attention level at the encoding phase was similar among pictures.

Another potential confound relates to the emotional valence of the subsequently remembered social pictures compared with the subsequently forgotten ones. Indeed, the mPFC activation could reflect a greater ratio of socioemotional pictures in the subsequently remembered subgroup. This is not the case because both subsequently remembered and subsequently forgotten social pictures had a proportion of $66 \%$ of socioemotional pictures. Similarly, one could argue that the social emotional pictures were perceived as being more emotional compared with the nonsocial emotional pictures. There was indeed a significant difference with respect to the emotional rating score between the social negative and the nonsocial negative pictures [68 vs. $91 ; t(16)=$ $5.77, p<.01]$. However, there was no significant difference between the social positive and the nonsocial positive pictures [261 vs. $239 ; t(16)=1.19, p>.05$ ] Moreover, a pilot study involving different subjects did not find such a difference in emotional rating between our social and nonsocial emotional pictures. Indeed, 12 healthy individuals used an ordinal scale ranging from 1 (very negative) to 7 (very positive) to rate the emotional valence of each picture presented on a computer monitor. Again, there was no significant difference between the social positive and the nonsocial positive pictures (5.66 vs. 5.83, $t=0.85, d f=11, p>.05$ ) and no difference either between the social negative and the nonsocial negative pictures $[1.96$ vs. $2.08 ; t(11)=0.74$, $p>.05]$. Thus, although we cannot completely rule out the possibility that social emotional pictures were perceived as more emotional compared with nonsocial emotional pictures, we believe that it is unlikely that our results concerning the dorsomedial PFC were driven by such a small effect.

There is also a potential limitation associated with the orienting task used during the encoding phase. Subjects were explicitly requested to attend to the social aspects of the stimuli ("Is a human being present in the picture?") but not to their emotional aspects. One can wonder if a potential difference regarding the level of processing (explicit vs. implicit) of these two types of content may partly explain the dorsomedial PFC modulation during the encoding of social content. Although this is possible, it is unlikely that the simple and quick identification of a human being in a picture will strongly promote complex social processing over and above emotional processing.

Finally, one problem with our recognition task was that some participants actually committed few mistakes. Even after the exclusion of five subjects who committed the fewest mistakes, we nonetheless had to pool some conditions for the SME analyses to respect a minimum cutoff of 10 events (mistakes) per condition. Pooling conditions led us to test the social factor and the emotional factor separately. As a result, the social and emotional content were not directly compared with each other but compared with their respective baseline (nonsocial and neutral). The SME result suggesting that the $\mathrm{MPFC}$ is a key region for social encoding cannot be interpreted as a statistical difference between social and emotional content, that is, a specific involvement of this region for social SME compared with emotional SME. To explore the potential specificity of the dorsomedial PFC for the encoding of social content compared with emotional content, we conducted a fixed-effect analysis that allowed us to define eight independent conditions: (1) socioemotional hits, (2) socioemotional misses, (3) socioneutral hits, (4) socioneutral misses, (5) nonsocial emotional hits, (6) nonsocial emotional misses, (7) nonsocial neutral hits, and (8) nonsocial neutral misses. We were then able to calculate the triple interaction (Social Content $\times$ Emotional Content $\times$ Subsequent Memory) among the regions involved in the successful encoding of social pictures. Although we used a very liberal threshold and we did not find a significant three-way interaction in the exact same dorsomedial PFC peak voxel as the one highlighted by the SME analysis (8, 66,24 , vs. $10,56,32$ ), the results of this post hoc analysis suggest that the involvement of the dorsomedial PFC during memory formation may be specific to social content compared with emotional content. We anticipate that this result will be confirmed by future studies.

\section{Conclusion}

Specific areas of the brain support the process of memory formation depending on the characteristics of the to-be-encoded material (Macrae et al., 2004; Brewer, Zhao, Desmond, Glover, \& Gabrieli, 1998; Kelley et al., 1998; Wagner, Schacter, et al., 1998). Although the modulatory effects of emotional information on memory performance and brain activity have been extensively studied (Smith, Henson, Dolan, \& Rugg, 2004; Erk et al., 2003, 2005), only a limited amount of data concerning such modulatory influence of social information is available. Using an experimental design in which both social and emotional dimensions of stimulus content were systematically manipulated, we observed that the mPFC is modulated by social information and not significantly modulated by emotional information during successful memory encoding. This finding provides a heuristic explanation to the apparent heterogeneity of previous functional neuroimaging findings pertaining to $\mathrm{mPFC}$ involvement in memory encoding.

\section{Acknowledgments}

This work was supported by grants from the Natural Sciences and Engineering Research Council (Grant 238617) and the Canadian Institutes of Health Research (Grant 53280) and by scholarships to P.-O. Harvey from the Canadian Institutes of 
Health Research and the Fond de Recherche en Santé du Québec. We thank H. Sutton, M. Menear, S. Partridge, and Dr. J. Armony for helpful suggestions and A. Achim for technical advice. We thank S. Dubal for providing some stimuli.

Reprint requests should be sent to Martin Lepage, Brain Imaging Group, Douglas Hospital Research Centre, Frank B. Common (FBC) Pavilion, 6875 boulevard LaSalle, Verdun, Québec, Canada H4H 1R3, or via e-mail: martin.lepage@mcgill.ca.

\section{REFERENCES}

Achim, A. M., \& Lepage, M. (2005). Neural correlates of memory for items and for associations: An event-related functional magnetic resonance imaging study. Journal of Cognitive Neuroscience, 17, 652-667.

Adolphs, R. (1999). Social cognition and the human brain. Trends in Cognitive Sciences, 3, 469-479.

Adolphs, R., Tranel, D., \& Damasio, A. (1998). The human amygdala in social judgment. Nature, 393, 470-474.

Adolphs, R., Tranel, D., Damasio, H., \& Damasio, A. (1994). Impaired recognition of emotion in facial expressions following bilateral damage to the human amygdala. Nature, 372, 669-672.

Ashburner, J., \& Friston, K. (1997). Multimodal image coregistration and partitioning-A unified framework. Neuroimage, 6, 209-217.

Brewer, J. B., Zhao, Z., Desmond, J. E., Glover, G. H., \& Gabrieli, J. D. E. (1998). Making memories: Brain activity that predicts how well visual experience will be remembered. Science, 281, 1185-1187.

Buckner, R., Kelley, W., \& Petersen, S. (1999). Frontal cortex contributes to human memory formation. Nature Neuroscience, 2, 311-314.

Bush, G., Luu, P., \& Posner, M. (2000). Cognitive and emotional influences in anterior cingulate cortex. Trends in Cognitive Sciences, 4, 215-222.

Canli, T., Zhao, Z., Brewer, J., Gabrieli, J. D. E., \& Cahill, L. (2000). Event-related activation in the human amygdala associates with later memory for individual emotional experience. Journal of Neuroscience. 20. RC99.

Collins, D., Neelin, P., Peters, T., \& Evans, A. (1994). Automatic 3D intersubject registration of MR volumetric data in standardized Talairach space. Journal of Computer-Assisted Tomograpby, 18, 192-205.

Dolcos, F., LaBar, K., \& Cabeza, R. (2004a). Interaction between the amygdala and the medial temporal lobe memory system predicts better memory for emotional events. Neuron, 42, 855-863.

Dolcos, F., LaBar, K. S., \& Cabeza, R. (2004b). Dissociable effects of arousal and valence on prefrontal activity indexing emotional evaluation and subsequent memory: An event-related fMRI study. Neuroimage, 23, 64-74.

Erk, S., Kiefer, M., Grothe, J., Wunderlich, A., Spitzer, M., \& Walter, H. (2003). Emotional context modulates subsequent memory effect. Neuroimage, 18, 439-447.

Erk, S., Martin, S., \& Walter, H. (2005). Emotional context during encoding of neutral items modulates brain activation not only during encoding but also during recognition. Neuroimage, 26, 829-838.

Fletcher, P. C., \& Henson, R. N. A. (2001). Frontal lobes and human memory: Insights from functional neuroimaging. Brain, 124, 849-881.

Fossati, P., Hevenor, S., Lepage, M., Graham, S., Grady, C., Keightley, M., et al. (2004). Distributed self in episodic memory: Neural correlates of successful retrieval of self-encoded positive and negative personality traits. Neuroimage, 22, 1596-1604

Fossati, P., Hevenor, S. J., Graham, S. J., Grady, C. Keightley, M. L., Craik, F., et al. (2003). In search of the emotional self: An fMRI study using positive and negative emotional words. American Journal of Psvchiatrv 160, 1938-1945.

Gallagher, H. L., \& Frith, C. D. (2003). Functional imaging of 'theory of mind.' Trends in Cognitive Sciences, 7 , $77-83$.

Gallese, V., \& Goldman, A. (1998). Mirror neurons and the simulation theory of mind-reading. Trends in Cognitive Sciences, 2, 493-501.

Geday, J., Gjedde, A., Boldsen, A., \& Kupers, R. (2003). Emotional valence modulates activity in the posterior fusiform gyrus and inferior medial prefrontal cortex in social perception. Neuroimage, 18, 675-684.

Habib, R., Nyberg, L., \& Tulving, E. (2003). Hemispheric asymmetries of memory: The HERA model revisited. Trends in Cognitive Sciences, 7, 241-245.

Hamann, S., Ely, T., Grafton, S., \& Kilts, C. (1999). Amygdala activity related to enhanced memory for pleasant and aversive stimuli. Nature Neuroscience, 2, 289-293.

Iacoboni, M., Lieberman, M. D., Knowlton, B. J., MolnarSzakacs, I., Moritz, M., Throop, C. J., et al. (2004). Watching social interactions produces dorsomedial prefrontal and medial parietal BOLD fMRI signal increases compared to a resting baseline. Neuroimage 21, 1167-1173.

Kao, Y., Davis, E., \& Gabrieli, J. (2005). Neural correlates of actual and predicted memory formation. Nature Neuroscience, 8, 1776-1783.

Kelley, W., Miezin, F., McDermott, K., Buckner, R., Raichle, M., Cohen, N., et al. (1998). Hemispheric specialization in human dorsal frontal cortex and medial temporal lobe for verbal and nonverbal memory encoding. Neuron, 20, 927-936.

Kelley, W. M., Macrae, C. N., Wyland, C. L., Caglar, S., Inati, S., \& Heatherton, T. F. (2002). Finding the self? An event-related fMRI study. Journal of Cognitive Neuroscience, 14, 785-794.

Lang, P., Greenwald, M., Bradley, M., \& Hamm, A. (1993). Looking at pictures: Affective, facial, visceral, and behavioral reactions. Psychopbvsiologv 30, 261-273.

Laviolette, S. R., Lipski, W. J., \& Grace, A. A. (2005). A subpopulation of neurons in the medial prefrontal cortex encodes emotional learning with burst and frequency codes through a dopamine D4 receptor-dependent basolateral amygdala input. Journal of Neuroscience. 25, 6066-6075.

Macrae, C. N., Moran, J. M., Heatherton, T. F., Banfield, J. F., \& Kelley, W. M. (2004). Medial prefrontal activity predicts memory for self. Cerebral Cortex. 14, 647-654.

McGaugh, J., McIntyre, C., \& Power, A. (2002). Amygdala modulation of memory consolidation: Interaction with other brain systems. Neurobiology of Learning and Memorv, 78, 539-552.

Mitchell, J. P., Macrae, C. N., \& Banaji, M. R. (2004). Encoding-specific effects of social cognition on the neural correlates of subsequent memory. Journal of Neuroscience, 24, 4912-4917.

Mitchell, J. P., Macrae, C. N., \& Banaji, M. R. (2005). Forming impressions of people versus inanimate objects: Social-cognitive processing in the medial prefrontal cortex. Neuroimage, 26, 251-257.

Norris, C. J., Chen, E. E., Zhu, D. C., Small, S. L., \& Cacioppo, J. T. (2004). The interaction of social and emotional 
processes in the brain. Iournal of Cognitive Neuroscience. 16, 1818-1829.

Paller, K., \& Wagner, A. (2002). Observing the transformation of experience into memory. Trends in Cognitive Sciences, 6, 93-102.

Phan, K., Wager, T., Taylor, S., \& Liberzon, I. (2002). Functional neuroanatomy of emotion: A meta-analysis of emotion activation studies in PET and fMRI. Neuroimage. 16, 331-348.

Rolls, E. (1996). The orbitofrontal cortex. Philosophical Transactions of the Royal Society of London, Series B, Biological Sciences, 351, 1433-1443; discussion $1443-1444$

Schilbach, L., Wohlschlaeger, A., Kraemer, N., Newen, A., Shah, N., Fink, G., et al. (2005). Being with virtual others: Neural correlates of social interaction. Neuropsychologia 44, 718-730.

Sergerie, K., Lepage, M., \& Armony, J. L. (2005). A face to remember: Emotional expression modulates prefrontal activity during memory formation. Neuroimage, 24, 580-585.

Smith, A., Henson, R., Dolan, R., \& Rugg, M. (2004). fMRI correlates of the episodic retrieval of emotional contexts. Neuroimage, 22, 868-878.

Stone, V. E., Baron-Cohen, S., \& Knight, R. T. (1998). Frontal lobe contributions to theory of mind. Lournal of Cognitive Neuroscience, 10, 640-656.
Symons, C., \& Johnson, B. (1997). The self-reference effect in memory: A meta-analysis. Psychological Bulletin, 121, 371-394.

Tulving, E., Kapur, S., Craik, F., Moscovitch, M., \& Houle, S. (1994). Hemispheric encoding/retrieval asymmetry in episodic memory: Positron emission tomography findings. Proceedings of the National Academv of Sciences. U.S.A., 91, 2016-2020.

Vogeley, K., Bussfeld, P., Newen, A., Herrmann, S., Happe, F., Falkai, P., et al. (2001). Mind reading: Neural mechanisms of theory of mind and self-perspective. Neuroimage 14 , 170-181.

Wagner, A., Poldrack, R., Eldridge, L., Desmond, J., Glover, G., \& Gabrieli, J. (1998). Material-specific lateralization of prefrontal activation during episodic encoding and retrieval. NeuroReport, 9, 3711-3717.

Wagner, A. D., Schacter, D. L., Rotte, M., Koutstaal, W., Maril, A., Dale, A. M., et al. (1998). Building memories: Remembering and forgetting of verbal experiences as predicted by brain activity. Science, 281, 1188-1191.

Winston, J., Strange, B., O'Doherty, J., \& Dolan, R. (2002). Automatic and intentional brain responses during evaluation of trustworthiness of faces. Nature Neuroscience. 5, 277-283.

Yancey, S., \& Phelps, E. (2001). Functional neuroimaging and episodic memory: A perspective. Journal of Clinical and Experimental Neuropsychology, 23, 32-48. 


\section{This article has been cited by:}

1. Kristina Dahlgren, Charles Ferris, Stephan Hamann. 2020. Neural correlates of successful emotional episodic encoding and retrieval: An SDM meta-analysis of neuroimaging studies. Neuropsychologia 143, 107495. [Crossref]

2. Vladimir Kosonogov, José M. Martínez-Selva, Ginesa Torrente, Eduvigis Carrillo-Verdejo, Juan Sánchez-Navarro. 2020. Does Social Content Influence the Subjective Evaluation of Affective Pictures?. The Spanish Journal of Psychology 23. . [Crossref]

3. Sivaniya Subramaniapillai, Sricharana Rajagopal, Abdelhalim Elshiekh, Stamatoula Pasvanis, Elizabeth Ankudowich, M. Natasha Rajah. 2019. Sex Differences in the Neural Correlates of Spatial Context Memory Decline in Healthy Aging. Journal of Cognitive Neurosience 31:12, 1895-1916. [Abstract] [Full Text] [PDF] [PDF Plus]

4. Erik A. Wing, Vijeth Iyengar, Thomas M. Hess, Kevin S. LaBar, Scott A. Huettel, Roberto Cabeza. 2018. Neural mechanisms underlying subsequent memory for personal beliefs:An fMRI study. Cognitive, Affective, \& Behavioral Neuroscience 18:2, $216-231$. [Crossref]

5. Florin Dolcos, Yuta Katsumi, Mathias Weymar, Matthew Moore, Takashi Tsukiura, Sanda Dolcos. 2017. Emerging Directions in Emotional Episodic Memory. Frontiers in Psychology 8. . [Crossref]

6. Thomas M. Hess, Lauren E. Popham, Claire M. Growney. 2017. Age-Related Effects on Memory for Social Stimuli: The Role of Valence, Arousal, and Emotional Responses. Experimental Aging Research 43:2, 105-123. [Crossref]

7. Jooyoung Oh, Ji-Won Chun, Eunseong Kim, Hae-Jeong Park, Boreom Lee, Jae-Jin Kim. 2017. Aberrant neural networks for the recognition memory of socially relevant information in patients with schizophrenia. Brain and Behavior 7:1, e00602. [Crossref]

8. Nilgun Turkileri, Michiko Sakaki. Neural Mechanisms Underlying the Effects of Emotional Arousal on Memory 43-55. [Crossref]

9. Florin Dolcos, Yuta Katsumi, Ekaterina Denkova, Mathias Weymar, Sanda Dolcos. Current Issues and Emerging Directions in the Impact of Emotion on Memory: A Review of Evidence from Brain Imaging Investigations 57-101. [Crossref]

10. Thomaz Lüscher Dias, Hudson Fernandes Golino, Vinícius Elias Moura de Oliveira, Márcio Flávio Dutra Moraes, Grace Schenatto Pereira. 2016. c-Fos expression predicts long-term social memory retrieval in mice. Behavioural Brain Research 313, $260-271$. [Crossref]

11. Vladimir Kosonogov, Juan Pedro Sanchez-Navarro, Jose Maria Martinez-Selva, Ginesa Torrente, Eduvigis Carrillo-Verdejo. 2016. Social stimuli increase physiological reactivity but not defensive responses. Scandinavian Journal of Psychology 57:5, 393-398. [Crossref]

12. Hyung-Jun Yoon, Jin Seong Kim, Yu-Bin Shin, Soo-Hee Choi, Seung-Koo Lee, Jae-Jin Kim. 2016. Neural activity during selfreferential working memory and the underlying role of the amygdala in social anxiety disorder. Neuroscience Letters 627, $139-147$. [Crossref]

13. Monika Riegel, Małgorzata Wierzba, Anna Grabowska, Katarzyna Jednoróg, Artur Marchewka. 2016. Effect of emotion on memory for words and their context. Journal of Comparative Neurology 524:8, 1636-1645. [Crossref]

14. Carla Pais-Vieira, Erik A. Wing, Roberto Cabeza. 2016. The influence of self-awareness on emotional memory formation: an fMRI study. Social Cognitive and Affective Neuroscience 11:4, 580-592. [Crossref]

15. Carolina S. Makowski, Martin Lepage, Philippe-Olivier Harvey. 2016. Functional neural correlates of social approval in schizophrenia. Social Cognitive and Affective Neuroscience 11:3, 445-457. [Crossref]

16. Meghan W. Cody, Shari A. Steinman, Bethany A. Teachman. 2015. True and False Memories in Social Anxiety Disorder: Effects of Speech Anticipation and Social Content. Cognitive Therapy and Research 39:6, 797-807. [Crossref]

17. Elizabeth A. Kensinger, Angela Gutchess. Memory for Emotional and Social Information in Adulthood and Old Age 393-414. [Crossref]

18. Shijia Li, Riklef Weerda, Christopher Milde, Oliver T. Wolf, Christiane M. Thiel. 2014. Effects of acute psychosocial stress on neural activity to emotional and neutral faces in a face recognition memory paradigm. Brain Imaging and Bebavior 8:4, 598-610. [Crossref]

19. David Luck, Marie-Eve Leclerc, Martin Lepage. 2014. The Potentiation of Associative Memory by Emotions: An Event-Related FMRI Study. Advances in Neuroscience 2014, 1-9. [Crossref]

20. Laura K. Sasse, Matthias Gamer, Christian Büchel, Stefanie Brassen. 2014. Selective Control of Attention Supports the Positivity Effect in Aging. PLoS ONE 9:8, e104180. [Crossref]

21. Anjuli S. Bodapati, Ellen S. Herbener. 2014. The impact of social content and negative symptoms on affective ratings in schizophrenia. Psychiatry Research 218:1-2, 25-30. [Crossref]

22. Olivia A. Bjorkquist, Ellen S. Herbener. 2013. Social perception in schizophrenia: Evidence of temporo-occipital and prefrontal dysfunction. Psychiatry Research: Neuroimaging 212:3, 175-182. [Crossref] 
23. Ellen Greimel, Barbara Nehrkorn, Gereon R. Fink, Juraj Kukolja, Gregor Kohls, Kristin Müller, Martina Piefke, Inge KampBecker, Helmut Remschmidt, Beate Herpertz-Dahlmann, Kerstin Konrad, Martin Schulte-Rüther. 2012. Neural mechanisms of encoding social and non-social context information in autism spectrum disorder. Neuropsychologia 50:14, 3440-3449. [Crossref]

24. Mi Li, Ning Zhong, Kuncheng Li, Shengfu Lu. 2012. Functional activation of the parahippocampal cortex and amygdala during social statistical information processing. Cognitive Systems Research 17-18, 25-33. [Crossref]

25. Michiko Sakaki, Kazuhisa Niki, Mara Mather. 2012. Beyond arousal and valence: The importance of the biological versus social relevance of emotional stimuli. Cognitive, Affective, \& Behavioral Neuroscience 12:1, 115-139. [Crossref]

26. Roee Gilron, Angela H. Gutchess. 2012. Remembering first impressions: Effects of intentionality and diagnosticity on subsequent memory. Cognitive, Affective, \& Behavioral Neuroscience 12:1, 85-98. [Crossref]

27. David Maillet, M. Natasha Rajah. 2011. Age-related changes in the three-way correlation between anterior hippocampus volume, whole-brain patterns of encoding activity and subsequent context retrieval. Brain Research 1420, 68-79. [Crossref]

28. Vishnu P. Murty, Maureen Ritchey, R. Alison Adcock, Kevin S. LaBar. 2011. Reprint of: fMRI studies of successful emotional memory encoding: A quantitative meta-analysis. Neuropsychologia 49:4, 695-705. [Crossref]

29. Hongkeun Kim. 2011. Neural activity that predicts subsequent memory and forgetting: A meta-analysis of 74 fMRI studies. NeuroImage 54:3, 2446-2461. [Crossref]

30. Dorothée Feyers, Fabienne Collette, Arnaud D'Argembeau, Steve Majerus, Eric Salmon. 2010. Neural networks involved in selfjudgement in young and elderly adults. NeuroImage 53:1, 341-347. [Crossref]

31. Vishnu P. Murty, Maureen Ritchey, R. Alison Adcock, Kevin S. LaBar. 2010. fMRI studies of successful emotional memory encoding: A quantitative meta-analysis. Neuropsychologia 48:12, 3459-3469. [Crossref]

32. John David Bone. 2010. Irrational Capitalism: The Social Map, Neoliberalism and the Demodernization of the West. Critical Sociology 36:5, 717-740. [Crossref]

33. Angela H. Gutchess, Elizabeth A. Kensinger, Daniel L. Schacter. 2010. Functional neuroimaging of self-referential encoding with age. Neuropsychologia 48:1, 211-219. [Crossref]

34. Jacob Geday, Albert Gjedde. 2009. Attention, emotion, and deactivation of default activity in inferior medial prefrontal cortex. Brain and Cognition 69:2, 344-352. [Crossref]

35. Jacob Geday, Albert Gjedde. 2009. Monoaminergic modulation of emotional impact in the inferomedial prefrontal cortex. Synapse 63:2, 160-166. [Crossref]

36. Angela H. Gutchess, Allie Indeck. Cultural influences on memory 137-150. [Crossref]

37. Laura M. Holsen, Kim M. Dalton, Tom Johnstone, Richard J. Davidson. 2008. Prefrontal social cognition network dysfunction underlying face encoding and social anxiety in fragile X syndrome. NeuroImage 43:3, 592-604. [Crossref]

38. S. Vinogradov, T. L. Luks, B. J. Schulman, G. V. Simpson. 2008. Deficit in a Neural Correlate of Reality Monitoring in Schizophrenia Patients. Cerebral Cortex 18:11, 2532-2539. [Crossref]

39. H. Park, M. D. Rugg. 2008. The Relationship between Study Processing and the Effects of Cue Congruency at Retrieval: fMRI Support for Transfer Appropriate Processing. Cerebral Cortex 18:4, 868-875. [Crossref]

40. Angela H. Gutchess, Elizabeth A. Kensinger, Daniel L. Schacter. 2007. Aging, self-referencing, and medial prefrontal cortex. Social Neuroscience 2:2, 117-133. [Crossref] 